\title{
THE INFLUENCE OF THE DIPOL TRANSITION MATRIX ELEMENT ON THE XANES AND OPTICAL SPECTRA FOR CdTe*
}

\author{
R. Markowski, J. Oleszkiewicz, A. Kisiel \\ Department of Physics, Jagellonian University, Reymonta 4, 30-059 Kraków, Poland
}

The reflectivity and X-ray absorption near-edge spectra (XANES) for CdTe have been calculated with inclusion of the transition matrix element from band structure obtained from self-consistent linear muffin-tin-orbital (LMTO) method. The result has been compared with experimental data for the case of CdTe.

PACS numbers: 78.70.Dm, 78.20.Bh, 71.25.Tn

The bands and density of states have been obtained using the self-consistent, semirelativistic LMTO method [1, 2]. The LSDA potential was used in the form proposed by Vosko, Wilk and Nusair [3]. The openness of the zinc-blende structure was dealt in standard way by placing additional "empty spheres" into the unit cell. The calculations were carried out using the experimental lattice constant. The results of such band structure calculation formed a basis for X-ray absorption and optical spectra calculation. In the one-electron approximation the X-ray absorption coefficient from a core level with energy $E_{\mathrm{c}}$ to final unoccupied conduction states with energy $E_{f}^{k}$ is given by the expression

$$
\mu_{0}(\hbar \omega)=\text { const } \sum_{f} \int_{\mathrm{BZ}} \mathrm{d}^{3} k\left|M_{f \mathrm{c}}^{k}\right|^{2} \delta\left(E_{f}^{k}-E_{\mathrm{c}}-\hbar \omega\right) .
$$

The final conduction states have finite lifetime, so the Müller correction has been taken into account [4]. The absorption coefficient has been finally convoluted with Gaussian and Lorentzian functions. The value of Lorentzian width $\Gamma_{\mathbf{L}}$ was optimized to obtain the best agreement of the theoretical spectra with the experimental ones in the surrounding of the absorption edge (about $8 \mathrm{eV}$ around the edge first inflection point).

*This work was supported in part by the Polish Central Research program CPBP 01.12. 
The optical absorption is proportional to the imaginary part of the dielectric function. In the limit of vanishing linewidth the interband transition $\epsilon_{2}^{b}(\omega)$ contribution of the dielectric function reads $[5,6]$

$$
\epsilon_{2}^{b}(\hbar \omega)=\frac{\text { const }}{\omega^{2}} \sum_{i} \sum_{f} \int_{\mathrm{BZ}} \mathrm{d}^{3} k\left|M_{f i}^{k}\right|^{2} \delta\left(E_{f}^{k}-E_{i}^{k}-\hbar \omega\right) .
$$

From comparison of the theoretical results with experimental data follows that the Cd $L_{1}$ and Te $L_{1}$ theoretical X-ray absorption edges results give almost equivalent good description of the experimental spectra. It means that in the case of $\mathrm{Cd} L_{1}$ and $\mathrm{Te} L_{1}$ the energy dependence of the transition matrix element is so weak, that the constant transition matrix approximation seems to be good enough. The worst disagreement between theory and experiment exists for $\mathrm{Cd} L_{3}$ edge. The Fig. 1 presents the results for the $\mathrm{Cd} L_{3} \mathrm{X}$-ray absorption edge. The $L_{2,3}$ edges

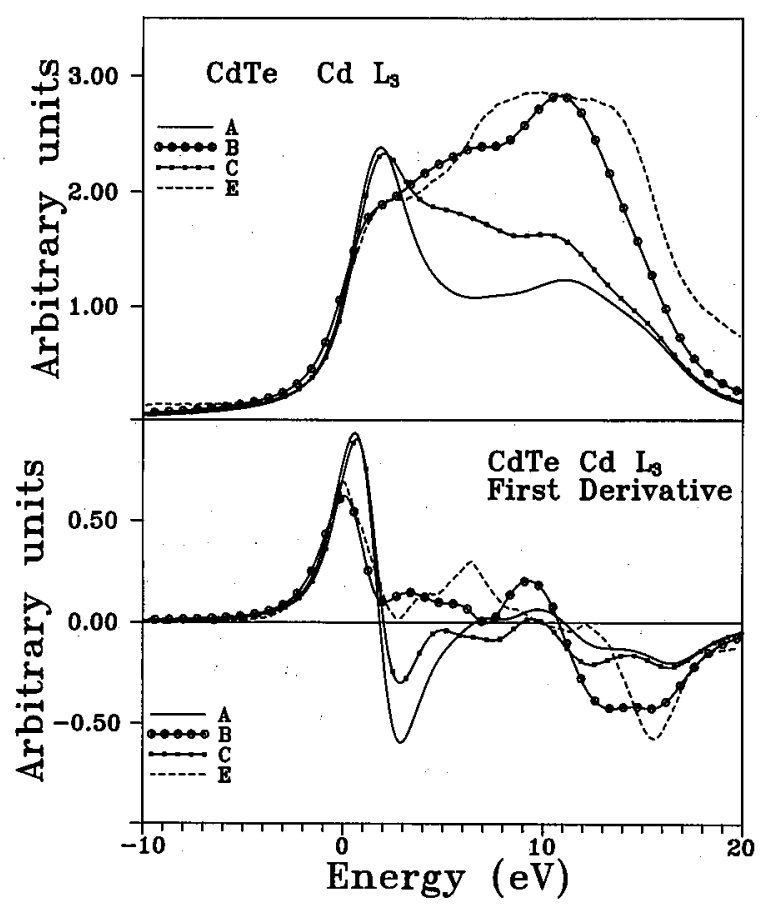

Fig. 1. The calculated $\mathrm{Cd} L_{3} \mathrm{X}$-ray edge spectra and their derivatives for CdTe. The curve A - the ratio $r_{c, s}: r_{\mathrm{c}, d}=1: 1$, the $r_{\mathrm{c}, s}=\left\langle M_{f \mathrm{c}}^{k}\right\rangle$. The curve B - the ratio was optimized to obtain the best agreement of theory with experiment in the area near the first inflection point of the edge (about $8 \mathrm{eV}$ around): $r_{\mathrm{c}, s}: r_{\mathrm{c}, d}=1: 3.3$. The curve $\mathrm{C}$ - the calculations with full inclusion of dipole transition matrix element.

arise from superposition of the transition to $s$-like and $d$-like conduction states. The constant matrix element approximation causes an additional problem of the value 
of the ratio between the matrix elements of $s$ - and $d$-like states (see Fig. 1). The inclusion of transition matrix element changes drastically the calculated shape, but still not enough. The main reason of it is the fact that the LMTO method uses finite basis function set. In the presented calculations, the basis function set includes the $4 d \mathrm{Cd}$ functions but does not include the $5 d \mathrm{Cd}$ functions. So, in order to use the LMTO method in future with XANES method, the LMTO basic function set should be enlarged. Such a modification of the LMTO method is not so easy, because the basis function set should contain the $4 d$ and $5 d$ Cd functions having the same crystal symmetry and that causes some additional computational problems.

The unadjusted absorption spectrum of CdTe is presented in the upper panel of Fig. 2. In order to illustrate the effects of the matrix elements we should compare

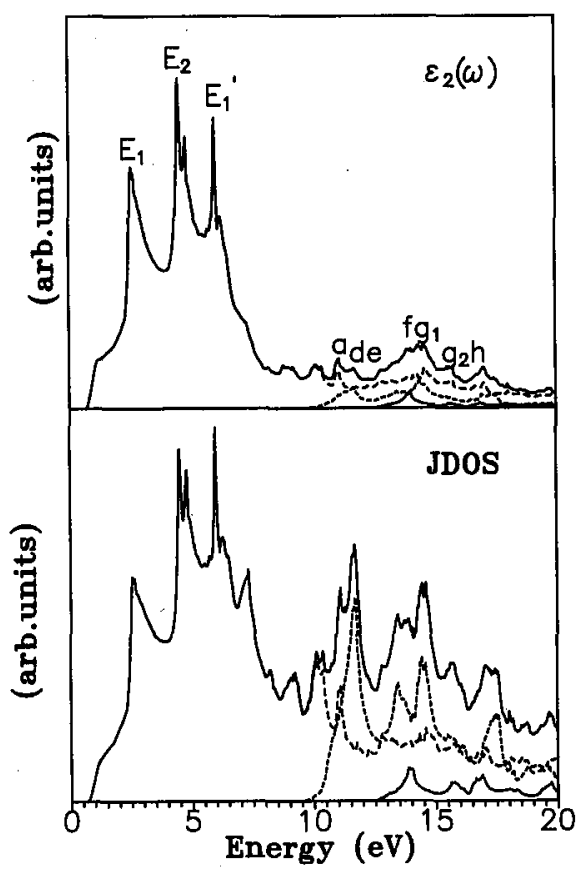

Fig. 2. Imaginary part of the dielectric function of CdTe (solid line, upper panel) and joint density of states divided by $\omega^{2}$ (solid line, lower panel). Short-dashed line represents the first bands contribution. Medium-dashed line the 2-6 bands contribution. Long-dashed line the 7-9 bands contribution.

the imaginary part of the dielectric function $\varepsilon_{2}^{b}(\omega)$ calculated with inclusion of the transition matrix elements and the joint density of states function divided by $\omega^{2}$ (Fig. 2). The peaks present in the JDOS at the UV energy range are reduced significantly in the $\epsilon_{2}^{b}(\omega)$ spectrum because the transition probability becomes small at high energy. Contrary to the common belief, apart from the strong contribution from the $\mathrm{Cd} 4 d$ states transitions, we find in this energy region a very significant 
contribution of the transitions originating in the upper part of the valence band to the higher conduction bands. In general, our calculations allow for a consistent assignment of the structures in the experimental reflectivity spectrum for CdTe [5]. A quantitative comparison to the experiment is only meaningful if the matrix elements of $\boldsymbol{P}$ operator are included in the calculation of the $\epsilon_{2}^{b}(\omega)$ spectrum.

\section{References}

[1] O. K. Anderson, Phys. Rev. B 12, 3060 (1975).

[2] H.L. Skriver, The LMTO Method, Vol. 41 of Solid State Sciences, Springer, Berlin 1984.

[3] S.H. Vosko, L. Wilk, M. Nusair, Can. J. Phys. 58, 1200 (1980).

[4] J.E. Müller, O. Jepsen, J.W. Wilkins, Solid State Commun. 42, 365 (1982).

[5] R. Markowski, M. Podgórny, J. Phys. Condens. Matter (1990) (accepted).

[6] Y.A. Uspenski, E.G. Maksimov, S.N. Rashkeer, I.I. Mazin, Z. Phys. B 53, 263 (1983).

[7] W. Speier, J.C. Fuggle, P. Durham, R. Zeller, R.J. Blake, P. Stern, J. Phys. C 21, 2621 (1988). 